\title{
Asymptomatic Cerebral Vasoconstriction after Carotid Artery Stenting
}

\author{
DC.H. Kang, (D). Roh, (D).A. Yeom, (D) S.H. Ahn, (D) M.G. Park, (D) K.P. Park, and (D).K. Baik
}

\begin{abstract}
BACKGROUND AND PURPOSE: Carotid artery stent placement is widely performed for treatment of carotid stenosis. The purpose of this study is to present our observations on cerebral vasoconstriction in ipsilateral anterior circulation during immediate poststenting angiography in patients with near-total occlusion of the proximal ICA.
\end{abstract}

MATERIALS AND METHODS: We retrospectively reviewed patient data from December 2008 to December 2018. There were 28 patients with carotid near-total occlusion. Two neuroradiologists reviewed the final cerebral angiographic finding of carotid artery stent placement to evaluate the presence of vasoconstriction or vasodilation.

RESULTS: A total of 28 patients with near-total occlusion (mean \pm standard deviation age, $69.0 \pm 6.5$ years; $92.9 \%$ male) were analyzed. Ten patients showed vasoconstriction in the treated territory, and 18 patients did not show vasoconstriction after carotid artery stenting. There were no statistically significant differences in comorbidity, frequency of symptomatic lesions, antiplatelet medication, mean procedure time, and initial NIHSS and baseline modified Rankin scale scores between the 2 groups. However, vasoconstriction is more likely to happen in patients with isolated territory from the contralateral anterior and posterior circulation (66.7\% in the isolated territory group and $12.5 \%$ in the not-isolated territory group; $P<.05)$. No headache or neurologic deficit was noted in all 10 patients with cerebral vasoconstriction.

CONCLUSIONS: Cerebral vasoconstriction may occur after carotid artery stenting more frequently than expected. It occurs more frequently in patients with near-total occlusion and with isolation of the cerebral circulation. A large-scale study is necessary to assess the clinical implications of cerebral vasoconstriction after carotid artery stenting.

ABBREVIATIONS: CAS = carotid artery stenting; CPP = cerebral perfusion pressure; HPS = hyperperfusion syndrome; NTO = near-total occlusion

arotid artery stenting (CAS) and carotid endarterectomy are widely performed for the treatment of carotid stenosis to improve cerebral perfusion and prevent stroke recurrence. Both the morphology of the stenotic vessel and hemodynamics of cerebral perfusion change after CAS. Some changes are immediately notable based on postprocedural angiographic finding, among which is cerebral vasoconstriction. Cerebral vasoconstriction cases after carotid endarterectomy have often been reported. ${ }^{1-6}$ In

Received August 13, 2019; accepted after revision December 1.

From the Departments of Radiology (C.H.K., J.R., J.A.Y., S.K.B.) and Neurology (S.H.A., M.G.P., K.P.P.), Pusan National University Yangsan Hospital, Pusan National University School of Medicine, Yangsan, Korea.

This work was supported by a 2-year research grant of Pusan National University.

Please address correspondence to Seung Kug Baik, MD, PhD, Department of Radiology, Research Institute for Convergence of Biomedical Science and

Technology, Pusan National University Yangsan Hospital, Pusan National University

School of Medicine, 20 Geumo-ro, Mulgeum-eup, Yangsan 50612, Korea; e-mail: skbaik9@gmail.com.

Indicates article with supplemental on-line tables.

http://dx.doi.org/10.3174/ajnr.A6385 contrast, cerebral vasoconstriction cases after CAS have rarely been reported. ${ }^{7-12}$ The purpose of this retrospective study is to present our observation on cerebral vasoconstriction in the ipsilateral anterior circulation during immediate poststenting angiography in patients with near-total occlusion (NTO) of the proximal ICA.

\section{MATERIALS AND METHODS Design and Patient Selection}

We retrospectively reviewed patient data from December 2008 to December 2018. A total of 361 stent placement procedures (in 286 patients) were performed in our hospital. Of these, there were 296 carotid stent placement procedures (in 221 patients) for carotid atherosclerotic stenosis and 28 patients with carotid NTO (Fig 1). NTO was defined as ipsilateral distal ICA less than the contralateral distal ICA, or ipsilateral distal ICA equal to or less than the ipsilateral external carotid artery. ${ }^{13}$

Two interventional neuroradiologists (S.K. Baik and J. Roh) reviewed the final cerebral angiographic finding of CAS to 


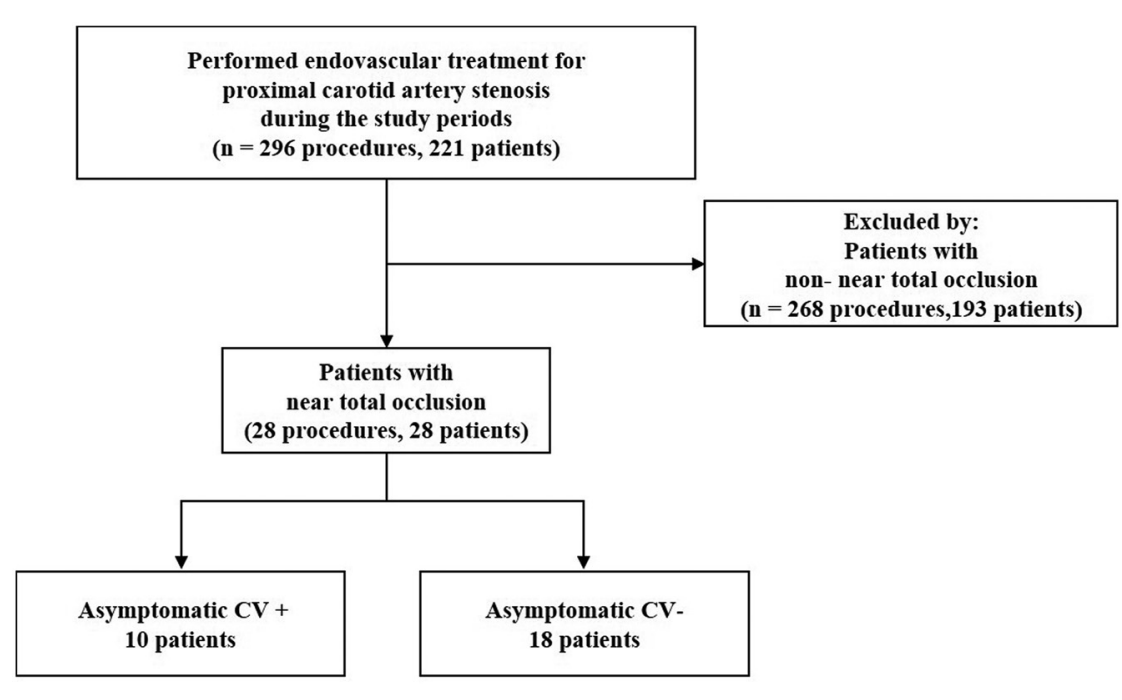

FIG 1. Patient flow diagram. CV indicates cerebral vasoconstriction; +, presence; -, absence.
The Guider Softip XF Catheter (Boston Scientific, Fremont, California) was used as a guide catheter in all patients, except in 2, in whom the Envoy Catheter (Codman \& Shurtleff, Raynham, Massachusetts) was used. Distal embolic protection devices were deployed in all patients except 1 . The types of embolic protection device were Spider FX (Covidien, Plymouth, Minnesota), FilterWire EZ (Boston Scientific, Natick, Massachusetts), and Emboshield NAV6 (Abbott Laboratories, Abbott Park, Illinois). We applied pre- and poststenting dilation in all the patients except 2 . Only prestenting balloon dilation was performed in 1 patient, and only poststenting balloon dilation was per-

evaluate the presence of vascular changes, such as vasoconstriction or vasodilation. Moreover, preprocedural DSA or MRA images were reviewed to determine if the vascular territory was isolated from the contralateral anterior or posterior circulation. Isolated circulation was defined as 1) signal intensity drop of the ipsilateral MCA-ICA territory, and 2) absence of an ipsilateral A1 segment and posterior communicating artery when evaluated on TOF-MRA. In 24 of the 28 patients (85.7\%), the results of independent review from the 2 radiologists (S.K. Baik and J. Roh) showed complete agreement, and the discrepancies in 4 patients were resolved after consensus meeting of the 2 neuroradiologists (S.K. Baik and J. Roh).

\section{Data Collection}

Demographic data and medical history (including age, sex, hypertension, diabetes mellitus, cigarette smoking, alcohol drinking, hyperlipidemia, atrial fibrillation, coronary artery disease, active cancer, and previous stroke) were collected. Procedural data collected were laterality, procedure time, deployed stents, balloon catheters, and embolic protection devices. In addition, data on the presence of symptoms caused by carotid stenosis, antiplatelet medication, plaque echogenicity on carotid Doppler ultrasonography, and initial NIHSS and baseline modified Rankin scale scores were collected. Moreover, data on the incidence of postprocedural parenchymal hemorrhage and discharge NIHSS and 3-month modified Rankin scale scores were collected.

\section{Procedural Technique}

All CAS procedures were performed by using a biplane angiography unit (Axiom Artis zee Biplane; Siemens, Erlangen, Germany). All the patients received aspirin (100 mg/day) and clopidogrel $(75 \mathrm{mg} /$ day $)$ treatment at least 7 days before the procedure. All procedures were performed with the patient under local anesthesia. A bolus of IV heparin $(50 \mathrm{IU} / \mathrm{kg}$ of body weight, 3000-5000 IU) was injected after the placement of the guide catheter. Subsequently, $1000 \mathrm{IU} / \mathrm{h}$ infusion was administered among all the patients. formed in another patient. The Ryujin balloon catheter (Terumo, Tokyo, Japan) and UltraSoft SV (Boston Scientific) were applied until 2011. Afterward, the Sterling balloon catheter (Boston Scientific) was used.

We applied open-cell type stents in all the patients and a combination of open- and closed-cell type stents in 1 patient. In most cases, Precise (Cordis, Fremont, California) was applied. However, in some cases, Protégé (Covidien), Acculink (Guidant, St. Paul, Minnesota), and Carotid Wallstent (Boston Scientific) were applied. After CAS, angiography of the proximal ICA and intracranial area and immediate postprocedural CT were performed in all patients.

\section{Statistical Analysis}

Statistical analysis was performed by using the Statistical Package for Social Sciences version 18.0 software for Windows (IBM, Armonk, New York). The patients were classified into 2 groups based on the presence of cerebral vasoconstriction. Univariate analysis was carried out by using the Mann-Whitney $U$ test for continuous variables and the Fisher exact test for categoric variables. A $P$ value of $<.05$ was considered as statistically significant.

\section{RESULTS}

The patients' baseline characteristics are shown in On-line Table 1. A total of 28 patients with NTO were analyzed. Of those, 26 patients $(92.9 \%)$ were men, and the mean \pm standard deviation age was $69.0 \pm 6.5$ years (range, $51-81$ years). The left ICA was treated in 15 patients $(53.6 \%)$, and 17 patients $(60.7 \%)$ had symptomatic stenosis. Ten patients showed multifocal vascular narrowing considered as vasoconstriction in the treated territory, whereas 18 patients did not show vasoconstriction after CAS. There were no statistically significant differences in comorbidity, frequency of symptomatic lesions, antiplatelet medication, mean procedure time, and initial NIHSS and baseline modified Rankin scale scores between the 2 groups. None of the 28 patients were taking vasoactive drugs.

However, vasoconstriction is more likely to happen in patients with isolated territory from the contralateral anterior 


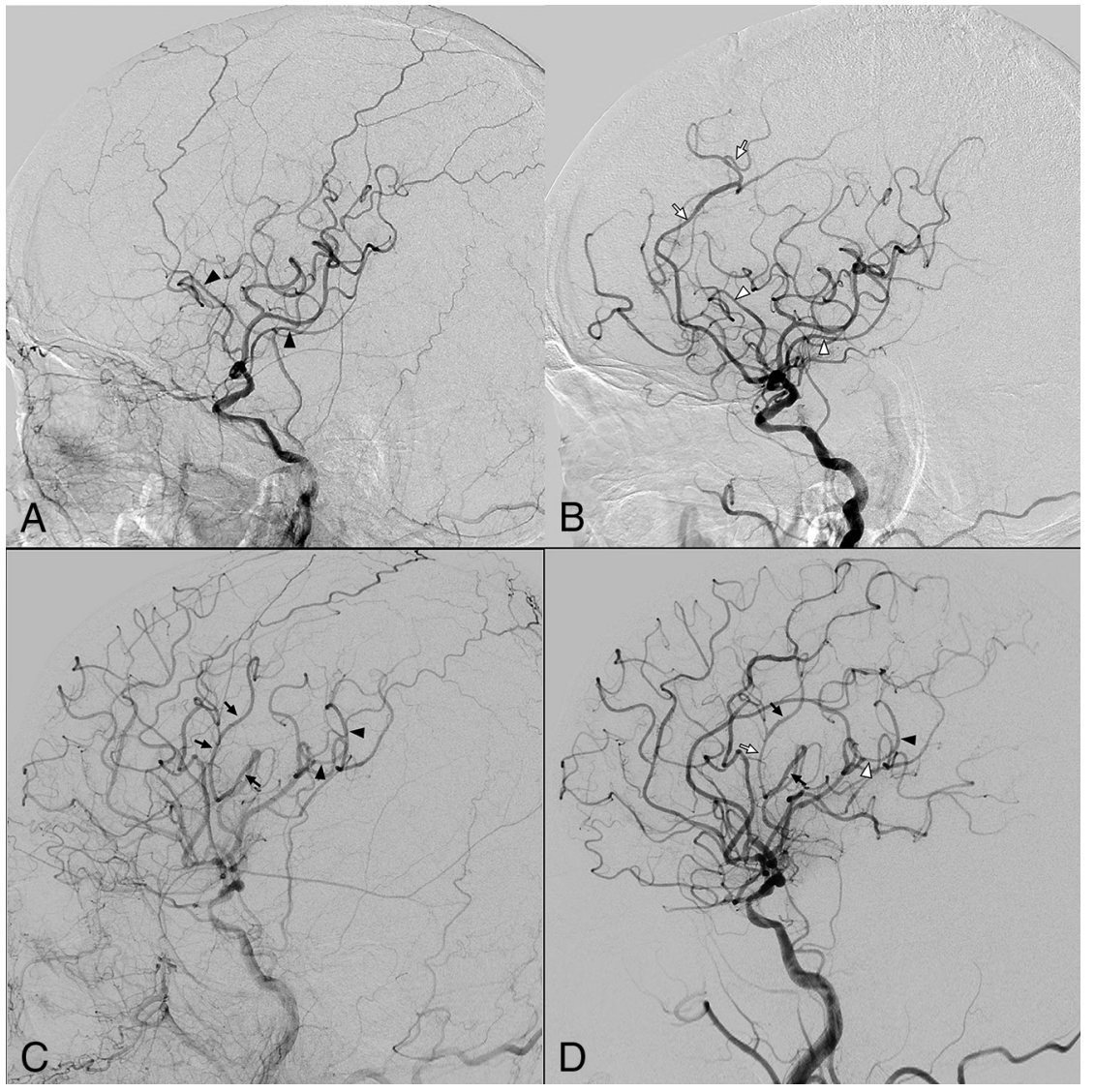

FIG 2. Two representative cases of vasoconstriction after revascularization therapy $(A$ and $B$, patient 10; $C$ and $D$, patient 24). $A$, On prestenting angiography, the distal branches of the left MCA are marked with black arrowheads, and the left ACA is invisible due to decreased cerebral blood flow. $B$, On poststenting angiography, these segments appear to be constricted (white arrowheads). The left ACA is visible, and the narrowed (believed to be constricted) segments are also observed in the left ACA. $C$ and $D, A$ superior division branch of the left MCA is marked with black arrows. C, An inferior division branch (arrowheads) on the angiography image before stent placement. $D$, On poststenting angiography, those branches are showing a narrowing and dilation appearance, and the narrowed (believed to be constricted) segments are marked with a white arrow and white arrowhead, respectively. Vasoconstriction phenomenon can be seen in arteries other than the marked branches. There is notable discrepancy of full/filling phase on pre- and poststenting angiography images, especially when compared with the external carotid artery filling. Note:-ACA indicates anterior cerebral artery.

and posterior circulation $(66.7 \%$ in the isolated territory group and $12.5 \%$ in the not-isolated territory group; $P<.05$ ). Vasoconstriction was observed in a larger number of the patients than expected (10/28 [35.7\%]). Small- and medium-sized vessels of the ipsilateral M2-3 and A2-3 were noted, and these changes were mostly multifocal. All the patients with vasoconstriction were asymptomatic.

The characteristics of 10 patients with asymptomatic cerebral vasoconstriction after CAS are shown in On-line Table 2. The characteristics of 18 patients without asymptomatic cerebral vasoconstriction after CAS are shown in On-line Table 3. There was no significant association between the types of embolic protection devices and cerebral vasoconstriction. No headache or neurologic deficit was noted in any of the 10 patients with cerebral vasoconstriction. There was no postprocedural hemorrhage in any of the 28 patients with NTO who underwent CAS, and no patient had a worsened NIHSS score by more than 2 points or worsened modified Rankin scale score at discharge. In addition, no patient had a decreased modified Rankin scale score at 90 days, except for 4 patients who were lost to follow-up. Cerebral vasoconstriction after CAS in 2 patients (cases 10 and 24) is shown in Fig 2.

\section{DISCUSSION}

There have been many reports of changes in hemodynamic status or cognitive function after CAS; however, few report the morphologic changes in intracranial vasculature after CAS. Morphologic changes of the intracranial artery were noted immediately after CAS in patients with NTO, and smooth vascular narrowing was observed. In our study, all the patients with vasoconstriction were asymptomatic, which indicated that these morphologic changes in the intracranial vasculature are physiologic responses to increased cerebral perfusion, which is a type of autoregulation.

Cerebral autoregulation is a homeostatic process that regulates and maintains constant $\mathrm{CBF}$ in a range of blood pressures. In healthy adults, $\mathrm{CBF}$ is constantly maintained between 50 and $150 \mathrm{~mm} \mathrm{Hg}$ cerebral perfusion pressure (CPP). The vascular adjustments that underlie autoregulation consist of constriction of cerebral resistance vessels when CPP increases and vasodilation of these vessels when CPP decreases. ${ }^{14}$ However, during cerebral ischemia, these mechanisms become dysfunctional and fail to compensate for CBF reduction. ${ }^{15}$

Moreover, autoregulation is impaired by stenosis or occlusion of the ICA. Two investigations by using transcranial Doppler sonography found that cerebral autoregulation is impaired in patients with severe ICA stenosis or occlusion. ${ }^{16,17}$ A correlation between the degree of stenosis and loss of autoregulation was noted in both groups. ${ }^{16,17}$ Of these, Reinhard et al ${ }^{17}$ found that cerebral autoregulation is most severely impaired in patients without Willisian collaterals. Furthermore, Haubrich et $\mathrm{al}^{18}$ demonstrated that impaired cerebral autoregulation may recover after CAS.

Compensatory mechanisms maintain cerebral perfusion in the initial stages of $\mathrm{CBF}$ reduction. Powers ${ }^{19}$ categorized these cerebrovascular adjustments into 3 stages: stage 0 , when CPP is normal; stage 1 , when $\mathrm{CPP}$ is reduced and autoregulation dilates the cerebral vessels to maintain CBF; and stage 2, when the compensatory capacity for cerebral vasodilation exceeds 


\begin{tabular}{|c|c|c|c|c|c|c|}
\hline Study & Age (y) & Sex & Laterality & Onset from CAS & Infarction & Outcome \\
\hline Arai et $\mathrm{al}^{7}$ & 72 & M & $\mathrm{R}$ & $13 \mathrm{~h}$ & Present & No deficit \\
\hline Shiraga et $\mathrm{al}^{12}$ & 62 & M & $\mathrm{R}$ & 1.5 days & Present & No deficit \\
\hline Soltanolkotabi et al ${ }^{11}$ & 49 & $\mathrm{~F}$ & $\mathrm{~L}$ & 14 days & Present & No deficit \\
\hline Aghaebrahim et $\mathrm{al}^{8}$ & 60 & M & $\mathrm{R}$ & $1 \mathrm{mo}$ & Not available & No deficit \\
\hline Watanabe et $\mathrm{al}^{9}$ & 74 & M & $\mathrm{R}$ & 17 days & Present & No deficit \\
\hline Higashi et al ${ }^{10}$ & 73 & M & $\mathrm{L}$ & Immediately & Present & Right hemiparesis, higher brain dysfunction \\
\hline
\end{tabular}

Note:-CV indicates cerebral vasoconstriction; $R$, right; $L$, left.

the limit, CBF begins to decrease, and cerebrovascular autoregulation is disrupted.

In patients with carotid NTO, CBF in the ipsilateral intracranial circulation is reduced. In response to CBF reduction, the cerebral vessels may be dilated to maintain cerebral perfusion. Likewise, autoregulation may be impaired in proportion to the severity of the stenosis. Immediately after CAS, a large amount of blood flows into the cerebral vessels, where autoregulation is impaired. We thought that cerebral vessels become uniformly constricted under normal autoregulation. Nevertheless, as in our patients, multifocal vasoconstriction may occur because of partial disruption of the autoregulation. Therefore, asymptomatic vasoconstriction in our patients is considered as a type of autoregulation or the boundary between autoregulation and pathologic condition. If the autoregulation is completely disrupted, then the cerebral vessels cannot constrict and are dilated. This condition is thought to be more vulnerable to hyperperfusion syndrome (HPS).

In our study, vasoconstriction occurred more frequently in patients with isolation of the cerebral circulation, which is consistent with the finding of Reinhard et al, ${ }^{17}$ that autoregulation is further impaired in patients without Willisian collaterals. This is probably because the cerebral hemodynamic reserve is further reduced, and autoregulation is subsequently further impaired in patients with isolation of the cerebral circulation. These changes are expected to return to normal when autoregulation is restored over time after CAS.

Six case reports of cerebral vasoconstriction after CAS have been published so far (Table). In these reports, cerebral vasoconstriction frequently occurred immediately after CAS and upto 1 month after CAS. In our study, we observed only the development of cerebral vasoconstriction immediately after CAS. In these 6 reports, the symptoms were mild in some patients and severe in the other cases.

However, in our study, all the patients with vasoconstriction were asymptomatic. This may be attributed to the location and degree of vasoconstriction, and susceptibility between individuals is different. Vasoconstriction after CAS has a broad spectrum of manifestations, depending on the severity of autoregulation impairment. If the autoregulation impairment is mild, then vasoconstriction is not prominent and the patients are asymptomatic. It may be a physiologic response, which is a type of autoregulation. Otherwise, if autoregulation impairment is severe, then vasoconstriction is prominent and may cause severe symptoms. It may be a pathologic condition, causing severe headache or stroke.

After CAS, impaired autoregulation of CBF also causes HPS. ${ }^{20}$ Both cerebral vasoconstriction and HPS are caused by abrupt increased blood flow into the cerebral vessels that are dilated by a compensatory response to severe carotid stenosis. Cerebral vasoconstriction probably occurs because the cerebral artery constricts abnormally. However, HPS probably occurs because the cerebral arteries cannot constrict and are dilated. Both are caused by impairment of cerebral autoregulation. To our knowledge, there have been no reports or studies on the morphologic changes of intracranial vessels in HPS after CAS.

After CAS, aside from HPS, cerebral vasoconstriction may occur. In the previous case report, the patient's symptom deterioration after CAS was misdiagnosed as HPS and antihypertensive medications were administered. Left MCA vasoconstriction was later observed. This caused severe disability in the patient. ${ }^{10}$ Therefore, when the patient's symptoms worsen after CAS, the possibility of cerebral vasoconstriction should be considered. It should be differentiated and treated appropriately. In previous reports, administration of calcium channel blockers, such as oral lomerizine hydrochloride ${ }^{10}$ or intra-arterial verapamil, ${ }^{8,11}$ was effective in the treatment of vasoconstriction after CAS. This study had some limitations. First, this was retrospective study. Second, the sample size was small.

\section{CONCLUSIONS}

Cerebral vasoconstriction may occur after CAS more frequently than expected. It occurs more frequently in patients with NTO and with isolation of the cerebral circulation. A large-scale study is necessary to assess the clinical implications of cerebral vasoconstriction after CAS.

Disclosures: Seung Kug Baik—RELATED: Grant: This work was supported by a 2year research grant from Pusan National University.

\section{REFERENCES}

1. Brick JF, Dunker RO, Gutierrez AR. Cerebral vasoconstriction as a complication of carotid endarterectomy. J Neurosurg 1990;73:15153 CrossRef Medline

2. Lopez-Valdes E, Chang HM, Pessin MS, et al. Cerebral vasoconstriction after carotid surgery. Neurology 1997;49:303-04 CrossRef Medline

3. Rosenbloom MH, Singhal AB. CT angiography and diffusion-perfusion MR imaging in a patient with ipsilateral reversible cerebral vasoconstriction after carotid endarterectomy. AJNR Am J Neuroradiol 2007;28:920-22 Medline

4. Wu TY, Frith RW, Barber PA. Reversible cerebral vasoconstriction following carotid endarterectomy. J Clin Neurosci 2011;18:1725-28 CrossRef Medline

5. Causey MW, Amans MR, Han S, et al. Reversible cerebral vasoconstriction syndrome is a rare cause of stroke after carotid endarterectomy. J Vasc Surg 2016;64:1847-50 CrossRef Medline

6. Fitas D, Carvalho M, Castro $\mathrm{P}$, et al. Cerebral vasoconstriction after carotid endarterectomy. Pract Neurol 2018;18:378-81 CrossRef Medline 
7. Arai M, Kuwayama N, Koide K, et al. Transient right hemisphere hypoperfusion following right carotid artery stenting: a case report (in Japanese). NOUSHINKEI KEKKANNAI TIRYOU 2013;7:119-26 CrossRef

8. Aghaebrahim A, Jadhav AP, Saeed Y, et al. Reversible cerebral vasoconstriction syndrome following carotid stenting. Neurology 2014; 83:570-71 CrossRef Medline

9. Watanabe $Y$, Takechi A, Kajiwara Y, et al. Reversible cerebral vasoconstriction syndrome following carotid artery stenting: a case report. Jpn J Stroke 2017;39:299-303 CrossRef

10. Higashi E, Uwatoko T, Mizokami T, et al. Reversible cerebral vasoconstriction complicated with brain infarction immediately after carotid artery stenting. Interv Neuroradiol 2018;24:688-92 CrossRef Medline

11. Soltanolkotabi M, Ansari S, Patel B, et al. Reversible cerebral vasoconstriction syndrome following carotid artery stenting. J NeuroIntervent Surg 2012;4:A49-A50 CrossRef

12. Shiraga S, Akai T, Takata $H$, et al. Possible cerebral infarction due to multiple segmental cerebral vasospasms after carotid artery stenting: a case report [in Japanese]. No Shinkei Geka 2015;43:108189 CrossRef Medline

13. Fox AJ, Eliasziw M, Rothwell PM, et al. Identification, prognosis, and management of patients with carotid artery near occlusion. AJNR Am J Neuroradiol 2005;26:2089-94 Medline
14. Kontos HA, Wei EP, Navari RM, et al. Responses of cerebral arteries and arterioles to acute hypotension and hypertension. Am J Physiol 1978;234:H371-83 CrossRef Medline

15. Langfitt TW, Weinstein JD, Kassell NF. Cerebral vasomotor paralysis as a cause of brain swelling. Trans Am Neurol Assoc 1964;89:21415 Medline

16. Gooskens I, Schmidt EA, Czosnyka M, et al. Pressure-autoregulation, $\mathrm{CO} 2$ reactivity and asymmetry of haemodynamic parameters in patients with carotid artery stenotic disease. A clinical appraisal. Acta Neurochir (Wien) 2003;145:527-32; discussion 532 CrossRef Medline

17. Reinhard M, Müller T, Roth M, et al. Bilateral severe carotid artery stenosis or occlusion - cerebral autoregulation dynamics and collateral flow patterns. Acta Neurochir (Wien) 2003;145:1053-60; discussion 1059-60 CrossRef Medline

18. Haubrich C, Kruska W, Diehl RR, et al. Recovery of the blood pressurecerebral flow relation after carotid stenting in elderly patients. Acta Neurochir (Wien) 2007;149:131-36; discussion 137 CrossRef Medline

19. Powers WJ. Cerebral hernodynamics in ischemic cerebrovascular disease. Ann Neurol 1991;29:231-40 CrossRef Medline

20. Buhk JH, Cepek L, Knauth M. Hyperacute intracerebral hemorrhage complicating carotid stenting should be distinguished from hyperperfusion syndrome. AJNR Am J Neuroradiol 2006;27:1508-13 Medline 